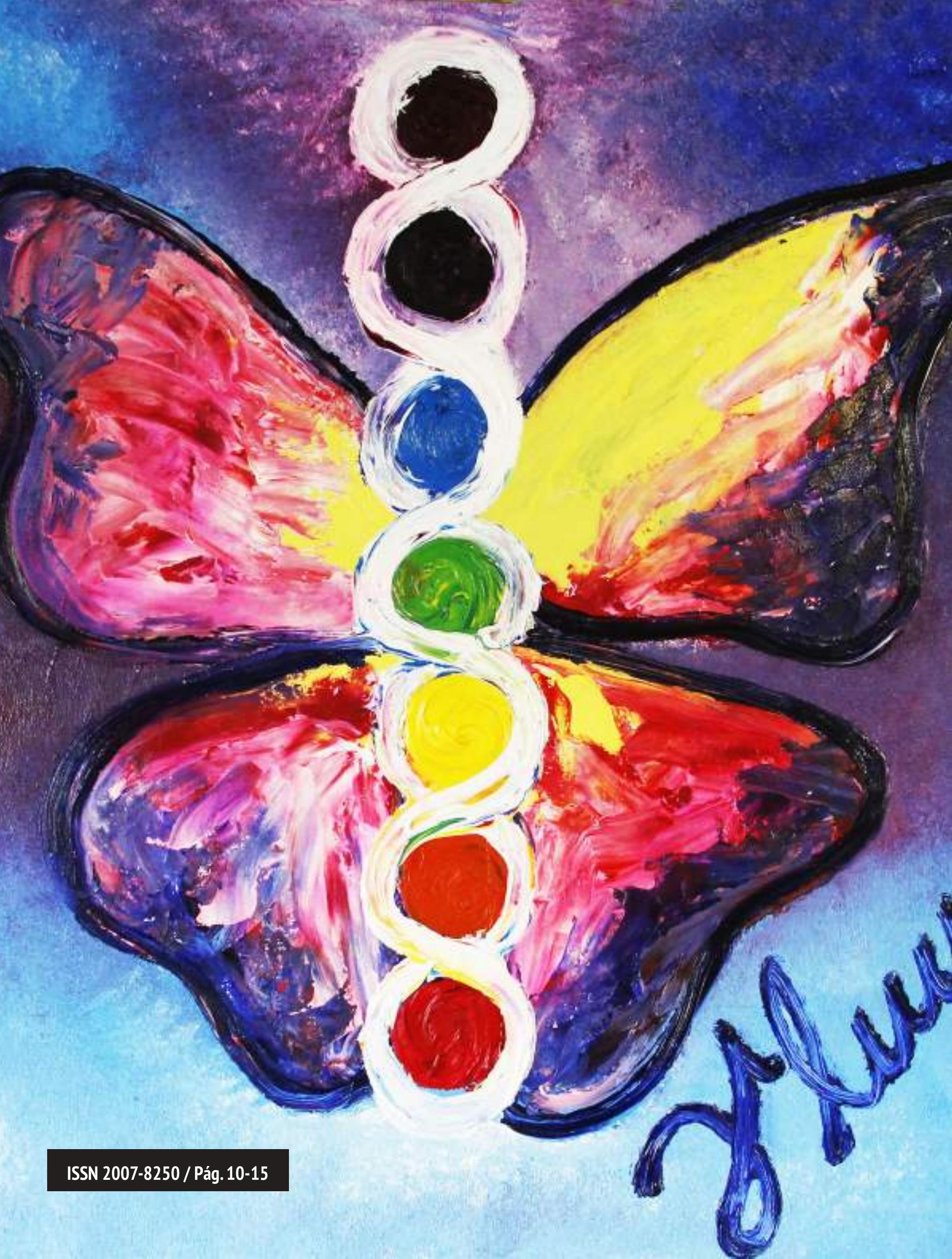




\section{NÚCLEO DE DESARROLLO ACADÉMICO: ACTITUD HACIA EL APRENDIZAJE}

Carlos Díaz Esparza

No. 2 - Septiembre 1990

$T$ no de los fenómenos que inquietan con frecuencia a los profesores, padres de familia, administradores educativos y la sociedad es la actitud del alumno hacia el estudio. 
e ha dado el nombre de estudiante a aquél cuyo rol consiste en estudiar, sin embargo, no siempre la conducta que se espera es la que presenta.

En estas líneas se intenta hacer un breve análisis acerca del tema, no se pretende agotarlo, no es un estudio exhaustivo, sólo es un conjunto de ideas cuya finalidad es aportar algunos datos al respecto. Inicialmente se propone una definición de actitud, posteriormente se plantean algunas características de ésta, después se mencionan sus dimensiones. Más adelante se aborda el tema central de las actitudes hacia el estudio y finalmente se presentan las conclusiones. ¿Qué es la actitud? No es fácil definirla, se han dado varios intentos, sin embargo no hay una sola definición. Con el fin de partir de una idea que permita ubicarse en el tema se propone la siguiente definición.

Actitud: "Tendencia o predisposición del individuo a evaluar en cierta forma un objeto o un símbolo del mismo...Las actitudes son el resultado de la forma en que se valora un objeto 0 un símbolo del mismo... entrañan sentimientos y emociones, creencias y acción”. (Lindgren 1978).

En cada actitud se pueden encontrar varios de estos elementos, aunque en algunas se presenta alguno de ellos en mayor grado que otros. Para cada objeto o símbolo se asume una actitud y el conjunto de estas integra el sistema de valores tanto del individuo como del grupo.

Las actitudes son el resultado de las experiencias y del aprendizaje derivado de ellas, que a su vez deter-

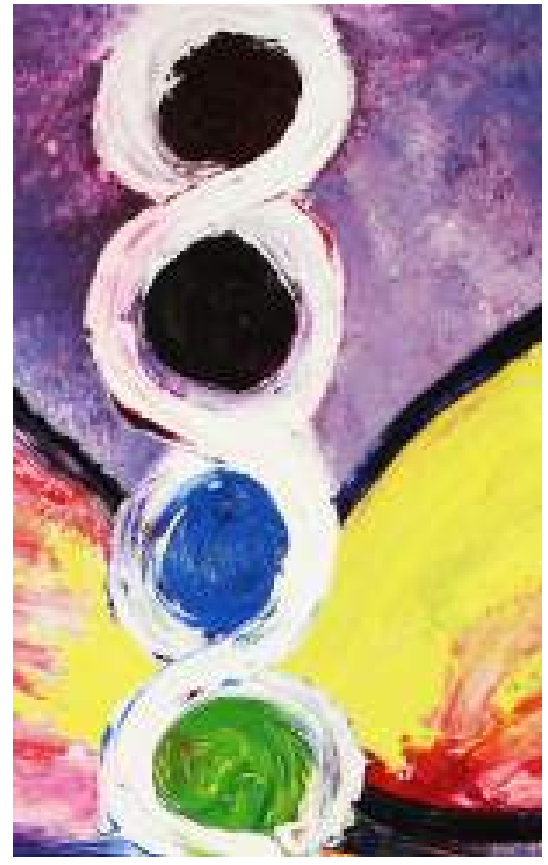

minan la percepción individual de la realidad. Con frecuencia las personas manifiestan conductas incongruentes debido a que tienen la "necesidad de satisfacer dos o más sistemas opuestos de creencias y valores" (Lindgren 1978).

No es raro encontrar declaraciones en las que las personas afirman tener una actitud, y cuando operacionalizan lo declarado actúan opuestamente a eso.

Las actitudes no pueden ser observadas directamente, se infieren de diversas manifestaciones conductuales; motoras y verbales.

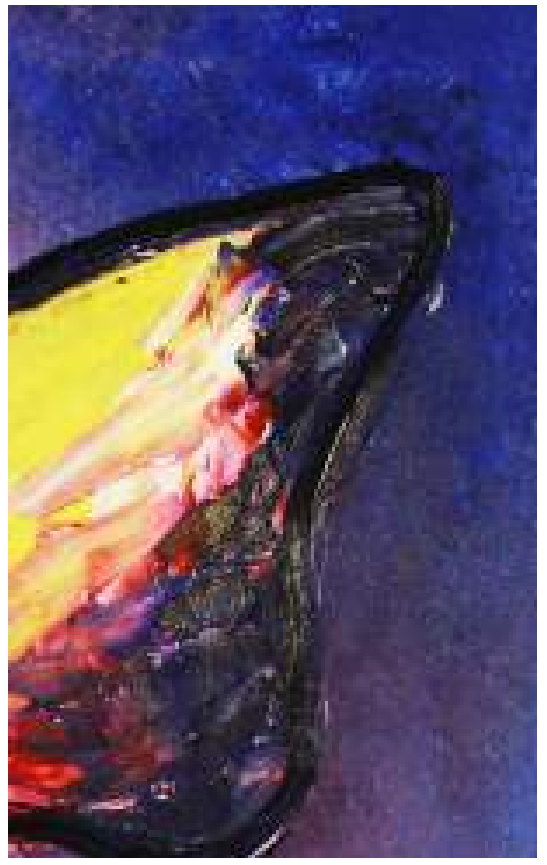

Lindgren (1978) propone 4 dimensiones de la actitud:

- Dirección. Redacción de acercamiento o alejamiento, aceptación 0 rechazo, agrado 0 desagrado que se adopta hacia un objeto, persona o situación.

- Intensidad. La fuerza con que se manifiesta la actitud.

- Centralidad. Ubicación en el sistema de actitudes y valores del individuo. Considerando como el centro a la persona y todo lo periférico se refiere a lo menos personal.

- Prominencia. Grado de importancia que tiene la actitud para la persona. 


\section{Las actitudes van siendo desarrolladas} por la persona conforme ésta va teniendo experiencias tanto consigo misma como con el medio externo (personas, objetos y situaciones)

Todas las actitudes, como se ha hecho notar, están bajo la influencia de sistemas de actitudes y valores generales propuestos por la comunidad en que vive el individuo.

Las actitudes van siendo desarrolladas por la persona conforme ésta va teniendo experiencias tanto consigo mismo como con el medio externo (personas, objetos, situaciones). Algunas desempeñan una función específica en cierta etapa del desarrollo personal, mientras otras van siendo reforzadas y mantenidas por largo plazo. Las actitudes hacia el estudio, como un caso específico se determinan a partir de diferentes variables.
Algunas de estas pueden ser:

1. Conocimiento de sí mismo en cuanto a habilidades y limitaciones

2. Padres

3. Profesores

4. Compañeros

5. Tipo de material (tarea) a mayor complejidad menos esfuerzo.

Autoconocimiento. El conocimiento de las habilidades personales es una variable que puede promover actitudes de rechazo, neutralidad 0 aceptación. Las experiencias vividas van proporcionando las bases para la emisión del comportamiento actitudinal por lo que es de esperarse

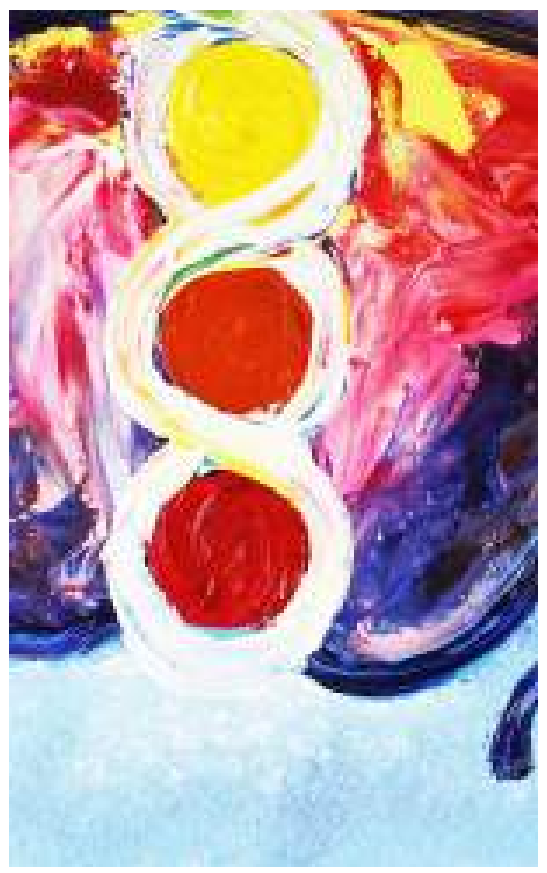

que cuando más autoconocimiento mayor congruencia se tendrán en las actitudes.

En base a la observación del comportamiento de los estudiantes, tanto de Licenciatura como de maestría de la $U$ de $M$ se considera que existe un autoconocimiento pobre relativo a sus habilidades, lo que se refleja en actitudes de rechazo hacia el estudio. Se ha encontrado que los estudiantes rechazan frecuentemente aquellos cursos en los que deben aplicar habilidades desconocidas por ellos 0 que consideran no poseer totalmente o forma parcial.

Esto lleva suponer que no existe una idea precisa acerca de las potencialidades, motivaciones y limitaciones personales.

Padres. Tanto los padres como otras personas llegan a tener un fuerte impacto sobre la conducta de los estudiantes debido a que tienden a ser modelos en los que se apoyan para el desarrollo de sus actitudes.

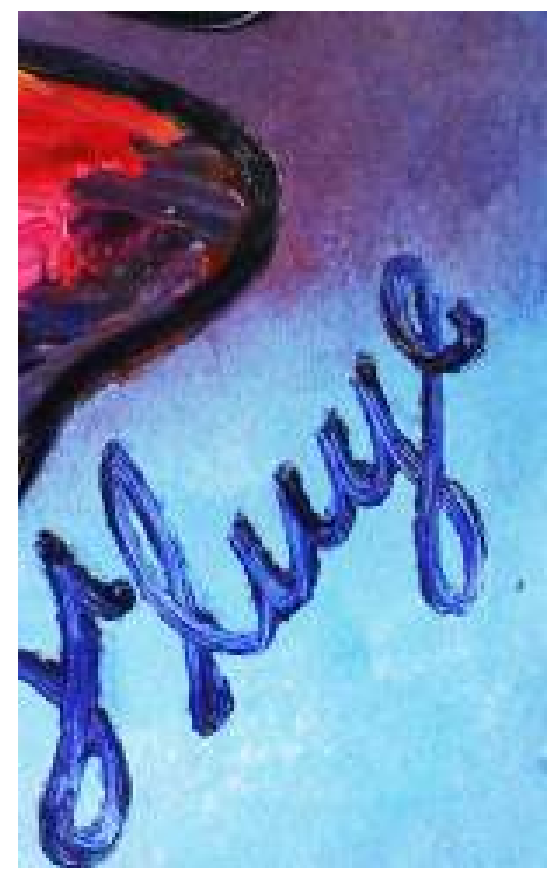


Si los padres valoran escasamente el estudio es más probable que los hijos asuman la misma actitud. A su vez si el valor que los padres le dan a las notas escolares es mayor que al estudio mismo, como medio de aprendizaje, se puede esperar que el hijo adopte una actitud similar.

Profesores. La influencia que estos tienen sobre las actitudes del alumno hacia el estudio se refleja con frecuencia en las opiniones de éstos.

A menudo se escucha el comentario, por parte de los estudiantes, del valor que les conceden al estudio como una consecuencia de la percepción que tienen de sus profesores.

El estudiante que adopta actitudes de aceptación puede ser aquél cuyos profesores los estimulan continuamente mediante el interés en sus avances en la materia, lo guían cuando se presentan dificultades que puedan estar fuera de sus posibilidades de resolución, manifiestan estar preparados en los temas de sus

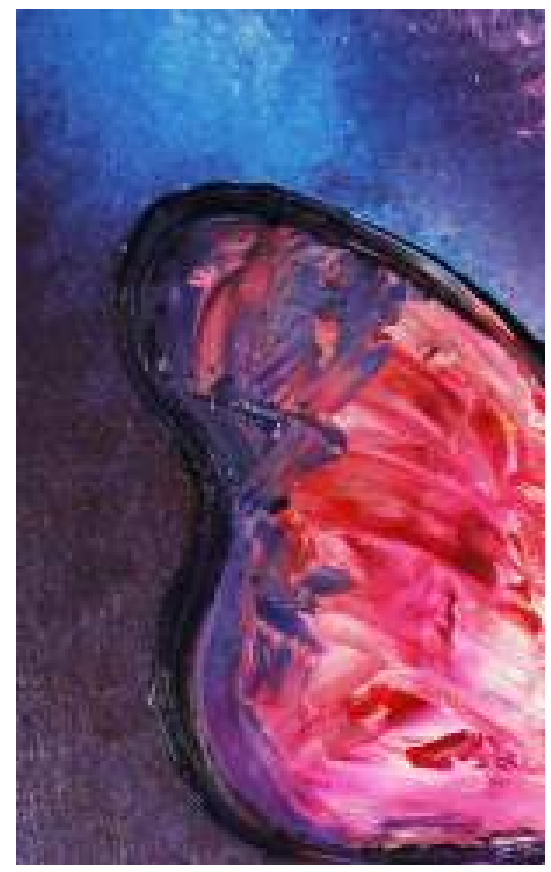

\section{El estudiante que adopta actitudes de aceptación puede ser aquél cuyos profesores lo estímulan continuamente mediante el interés en sus avances en la materia}

cursos como producto de una actualización continua, le dan valor positivo a su materia, les retroalimentan con frecuencia.

Compañeros. Los compañeros son otra fuente de actitudes ya que, dependiendo del interés que manifiestan hacia el estudio, genera una fuerte presión sobre el estudiante.

En muchos casos la tendencia a la conformidad con el grupo derivada de la influencia social se percute en las actitudes del estudiante debido a que trata de evitar quedar marginado del grupo, ser clasificado negativamente o convertirse en objeto de las

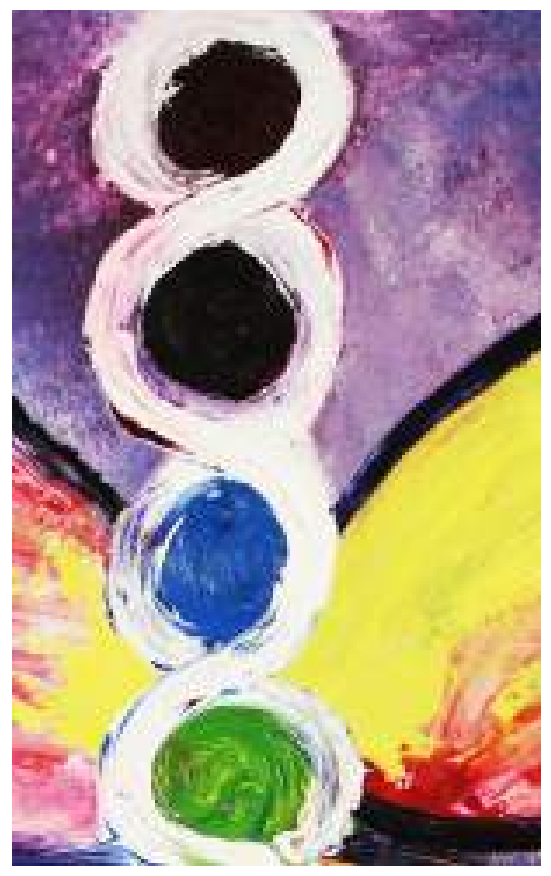

bromas y burlas de sus compañeros. La asociación con otro compañero tiene los mismos efectos antes mencionados. No sólo se dan actitudes negativas sino también positivas 0 neutras.

Condiciones Escolares. Son diversas las condiciones o factores escolares que promueven actitudes hacia el estudio, el tipo de material, la complejidad de la tarea, el contenido de cada curso, el apoyo bibliográfico y hemerográfico, las instalaciones físicas (aulas, biblioteca, laboratorios), las actividades extra-académicas. Calificaciones, exámenes. Dependiendo de las expectativas de cada persona así como de su capacidad de adaptación y de su madurez será el tipo de actitud a desarrollo.

Valoración Social de los Productos Educativos. En forma similar a las variables ya mencionadas el valor que le da la sociedad, particularmente la familia y la comunidad, a los productos educativos tiende a contribuir al desarrollo de las actitudes hacia el estudio.

\section{CONCLUSIÓN}

¿Por qué se ha abordado este tema? La finalidad que se persigue al realizar este escrito, como se mencionó en su principio, es aportar algunas 
ideas a los profesores para que de alguna manera intenten comprender el comportamiento de los estudiantes.

Las consecuencias de las actitudes ante el estudio con frecuencia son poco positivas. En ocasiones el profesor se siente desanimado o molesto por los resultados de sus alumnos en su curso, las reacciones de enojo de los estudiantes cuando les propone realizar alguna consulta bibliográfica, la apatía que se manifiesta en éstos para llegar a clase con el material preparado, en fin, un sinnúmero de conductas que provocan tensiones ble para el aprendizaje.

El profesor toma decisiones, así como asume actitudes ante sus alumnos, en base a sus percepciones de las conductas actitudinales que éstos emiten. Por tanto, puede esperarse que cuando más los comprenda mayor sea la probabilidad de promover los cambios de actitud hacia el estudio que redunden en un mayor aprendizaje y formación de los estudiantes.

\section{RESUMEN}

La actitud es la conducta aprendida mediante la cual se expresa la valorización que cada persona le da a los fenómenos internos y externos que que propician un clima poco favora-

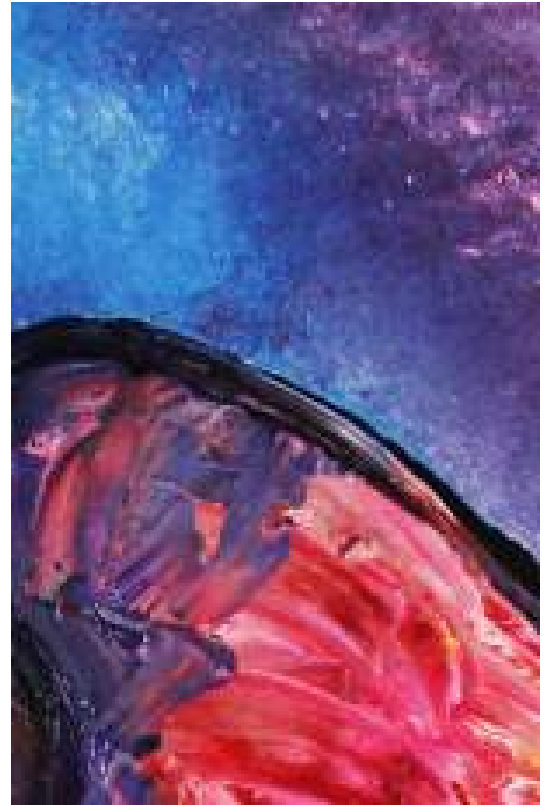

experimenta. Posee elementos conductuales, cognoscitivos, afectivos y axiológicos.

Las actitudes hacia el estudio son determinadas por muchas variables, no por una sola. Algunas de éstas son: autoconocimiento, autoconcepto; los padres, los profesores, los compañeros de la escuela, las condiciones escolares y la valoración social de los productos educativos.

Dependiendo del tipo de experiencia que viva la persona, de los elementos que participen en su desarrollo y de las variables que intervengan, se presentarán las actitudes positivas, neutras y negativas.

\section{Las actitudes hacia el estudio}

son determinadas por muchas

variables, no por una sola

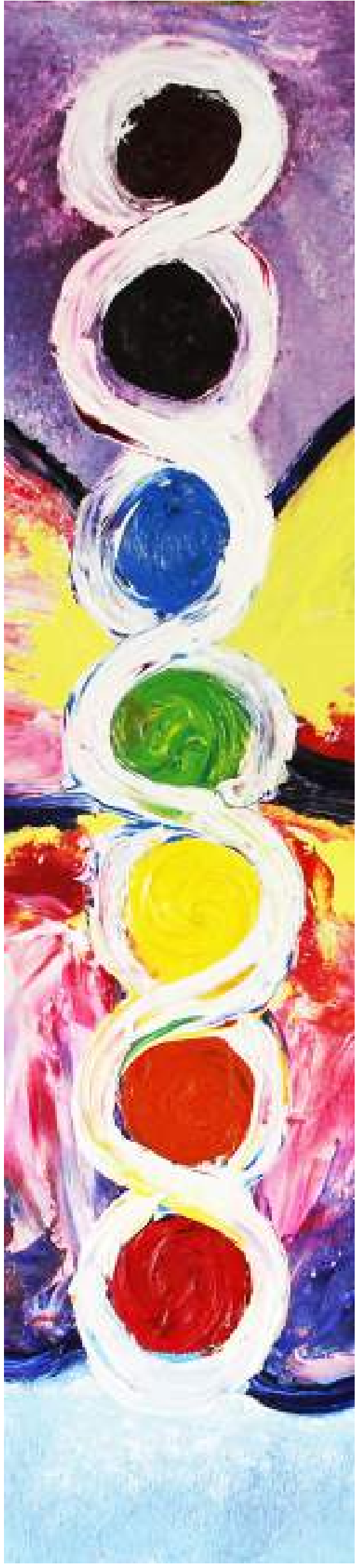

Presencia Universitaria 